\title{
IMPROVING MMI WITH ENHANCED-FCM FOR THE FUSION OF BRAIN MR AND SPECT IMAGES
}

\author{
Chung-Hsien Huang, Jiann-Der Lee \\ Department of Electrical Engineering, Chang Gung University \\ Tao-Yuan, Taiwan
}

\begin{abstract}
Recently: maximization mutual information (MMI) of image intensities has been proposed as a new matching criterion for automated multimodality image registration. However: the success of the MMI relies on the similarity of the histogram distribution between the images to be fused. This condition is usually hard to be achieved in practical application. Besides. MMI is time consuming hecause it needs to find an optimal solution about six parameters (three for shifts and three for rotations) during the registration process. To overcome these drawbacks of using traditional MMI, a novel scheme, named improved MMI, which is based on fuzzy c-means (FCM) and MMI, is proposed. The experimental results, using MR and SPECT images, to confirm the superior performance of the proposed method in comparison with the traditional MMI method are also included.
\end{abstract}

Biomed Eng Appl Basis Comm, 2004(August): 16: 185-189.

\section{INTRODUCTION}

In clinical applications, medical images obtained from various modalities usually provide valuable information of the patient for diagnosis. For example, functional images (PET, SPECT) provide metabolic or neuro-chemical changes characteristic, and anatomical images (CT, MRI) offer geometric and structural properties. SPECT image is a nuclear medicine technique in which temporal changes in the concentrations of radioactive tracers are recorded in brain and other target organs. It is a non-invasive nuclear medicine technique that utilizes radiopharmaccuticals and a tomography instrument to generate a quantitative image set. This functional imaging method is firmly established as important scientific and clinical research tool.

Received: Feb 4, 2004; Accepted: June 30, 2004

Correspondence: Jiann-Der Lee, Professor

Department of Electrical Engineering, Chang Gung University, Tao-Yuan, Taiwan 333

E-mail: jdlee@mail.cgu.edu.tw
Image fusion is a synthesized process, which combined multi-dimension information and displayed on a particular space, with the aim of providing either more condensed or more pertinent information. In general, using MMI [1-6] to register functional and anatomical image, physicians can get more diagnostic information on the focal part. However, it is note that the image intensity of MR corresponds to the hydrogen density of the body, and SPECT imaging corresponds to radioactive intensity but brain anatomical structure. Their histogram distributions are quite different, and this characteristic will cause MMI fall into a local maximum and result in the system failure. Clearly, it is mainly because traditional MMI has high correspondence with the joint MR-SPECT histogram. Moreover, $\mathrm{MMI}$ is also a time-consuming algorithm since it needs to derive an optimal solution for three translations and three rotations while registering two modality's spaces. To overcome these drawbacks of MMI, we first present an enhanced-FCM algorithm to generate the fuzzy map of brain tissue and then exclude the high radioactive intensity to replace the histogram of original image. Next, we find the ideal mid-sagittal plane of the brain such that the computing 
time required in the registration procedure can be reduced. Here, only three locating parameters ( 2 shifts and 1 rotations) are needed while applying the MMI on this mid-sagittal plane. Finally, by means of this scheme, the experimental results are shown that the registration and fusion of $M R$ and SPECT images can be performed fast and accurate.

The remainder of this paper is organized as follow. The proposed method for fusion of brain MR and SPECT images is described in section 2 . The experimental results to prove the effectiveness of our algorithm are shown in section 3. Finally, the conclusion is given in the last section.

\section{THE PROPOSED METHOD}

The whole flowchart of the proposed improved MMI scheme is illustrated in Fig. 1. In short, there are three steps in this scheme, which includes IMSP, Enhanced-FCM, and MMI algorithm. More specifically, IMSP algorithm [7] is employed to find the mid-sagittal plane of each image set. The aim of this step is to reduce a 3-D volume data to a 2-D image data. It can save the large computing cost for MMI. Next, by using Enhanced-FCM algorithm, the brain fuzzy map of each mid-sagittal plane is obtained. This fuzzy map can present brain histogram without noise and some needless information. Finally, MMI can get an accurate registration of MR and SPECT images.

\subsection{Ideal Mid-Sagittal Plane Algorithm}

IMSP algorithm is an edge-based, crosscorrelation approach that decomposes the plane-fitting problem into discovery of 2-D symmetry axes on each slice. The algorithm has strong tolerance to brain asymmetrics, input image offsets and image noise. By using IMSP, we can figure out the mid-sagittal planes of MR and SPECT images successfully. (as shown in Fig. 2).

The IMSP algorithm is briefly described as below:

Input : Brain image set of axial slice $\left(S_{1}, S_{2} \ldots S_{n}\right)$

Output : Rotation angle $\theta$

For $i=1$ to $\mathrm{n}$

Select Axial Image $S_{i}$

Construct the mirror image $S_{i}$ of $S_{i}$

Find the perpendicular bisector of images $S_{i}$ and $S_{i}$

For $\theta_{i}=-\theta$ to $\theta$

Find the maximum of cross correlation value of $S_{i}{ }^{\prime}$ and $\mathrm{S}_{\mathrm{i}}$ for each $\theta$

End

End angle $\theta_{i}$

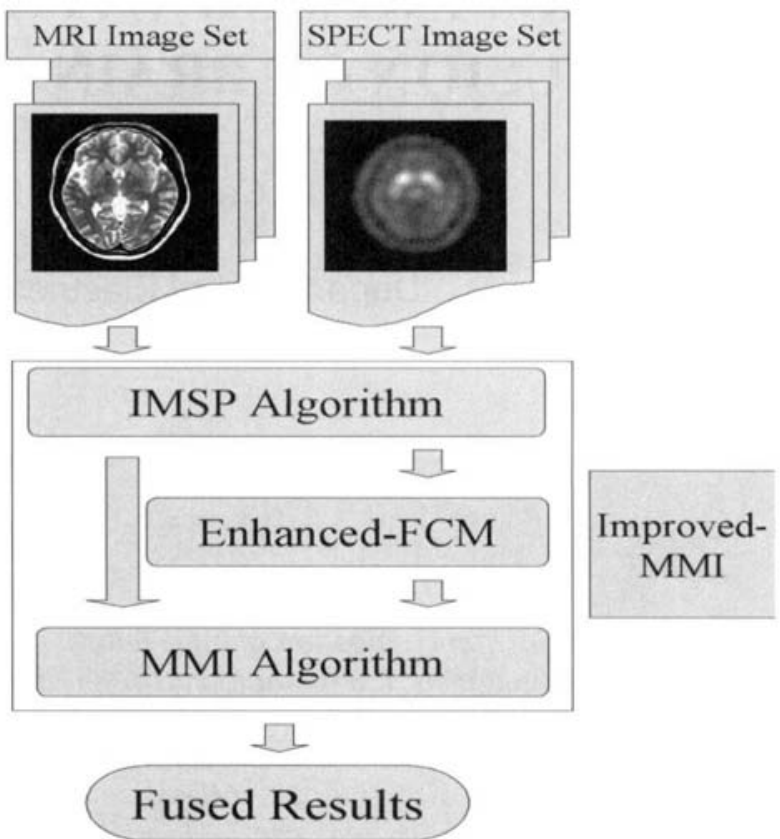

Fig. 1 The flowchart of the proposed improved MMI

\subsection{Enhanced Fuzzy C-Means Algorithm}

FCM is often used to image segmentation problem and has fine results [8-10]. It is a completely unsupervised method and convergence repeatedly, in order to find the optimum solution. The FCM algorithm, via fuzzy pixel classification, allows pixels belong to multiple classes with varying degrees of membership. The FCM algorithm for scalar data seeks the membership function $\mathbf{u}_{k(i, j)}$, i.e., Eq. (1) and the centroids $v_{k}$, i.e., Eq. (2), such that the objective function shown as Eq. (3) is minimized.

$$
\begin{aligned}
& J_{F C M}=\sum_{i, j \in n} \sum_{k=1}^{c} u_{k(i, j)}^{q}\left\|x(i, j)-v_{k}\right\|^{2} \\
& u_{k(1, j)}= {\left[\sum_{k=1}^{C}\left(\frac{\left\|x(i, j)-v_{k}\right\|}{\left\|x(i, j)-v_{n}\right\|}\right)^{2((q-1)}\right]^{-1}, 1 \leq k \leq c } \\
& v_{k}=\frac{\sum_{i, j \in n} u_{k(1, j)}^{q} \cdot x(i, j)}{\sum_{i, j \in n} u_{k(1, j)}^{q}}, 1 \leq k \leq c
\end{aligned}
$$

where $\mathbf{u}_{k(i, j)}$ is the membership value of the pixel $(i, j)$ in the $k$-th class, $v_{k}$ is the centroid of $k$-th class, and $q$ is a weighting exponent on each fuzzy membership and it determines the amount of 


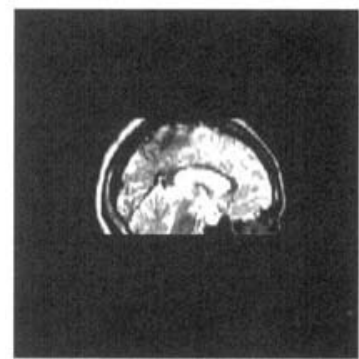

(a)MR image

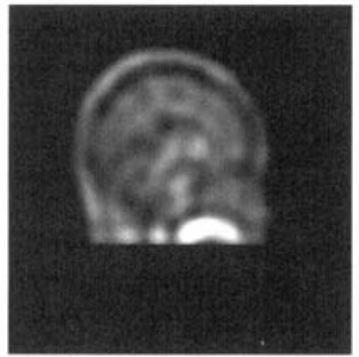

(b)SPECT image
Fig. 2 The mid-sagittal planes of MR and SPECT images

"fuzziness" of the resulting classification.By calculating Eq. (2) and Eq. (3) repeatedly, the objective value $J_{F C M}$ is converged on a static minimum by the way of the expectably accuracy with a preset threshold value. When the variation of $J_{F C M}$ is smaller than this threshold value, the process is terminated and the last $u_{k(i, j)}$ and $v_{k}$ are then used as the constraint parameters for segmentation.

Generally, the typical FCM chooses gray level of pixels in the image to be feature space. In accordance with the distribution of image's histogram, the FCM only considers the image intensity, but not the spatial relationship between a pixel and its neighbors. Therefore, FCM has weak resistance of various noises from the working environment. To overcome the weakness of typical FCM, we propose a novel improved-FCM method to increase the segmentation performance of brain MR and SPECT images. More specifically, a filter mask to represent the spatial relationship of pixel and its neighbors is employed in the converging process of FCM to achieve a more satisfied segmented result than previous FCM methods.

In the enhanced-FCM algorithm, $u_{k(i, j)}$ is the membership value of the pixel $(i, j)$ in the $k$-th class. And $\Delta i-\left\|x(i, j)-v_{k}\right\|$ denotes the distance or the variation degree between the pixel $\boldsymbol{x}(\boldsymbol{i}, \boldsymbol{j})$ and the centroid of $\boldsymbol{k}$-th class. Based on the reasonable concept that a candidate boundary pixel must have some degree of correlation with its neighbor pixels, we design a filter mask to redefine this pixel's feature $U$ as below

$U_{k(1, j)}=\frac{w_{1} u_{k(1-1, j-1)}+w_{2} u_{k(1, j-1)}+\ldots+w_{9} u_{k(1+1, j+1)}}{\left(w_{1}+w_{2}+\ldots+w_{9}\right)}$

Next, the new distance between a pixel and the centroid of a class is replaced with Eq. (5).

wherc $p$ is a modulated parameter ranging from 0

$$
\Delta F^{\prime}=\Delta F\left(1-p U_{k(i, j)}\right)
$$

to 1. It is noted that, when $p=0, \Delta F^{\prime}-\Delta F$ and Eq. (5) becomes the original FCM algorithm. That is, traditional FCM is a special case of Enhanced-FCM. In addition, it is obvious that a large $p$ denotes that the neighbor pixels have higher correlations with the center pixel. Therefore, we put the new distance function, i.e., Eq. (5), in the traditional FCM algorithm equations, i.e., Eq. (1) and Eq.(2), to replace the Euclidean distance function and run the same iterative calculation as FCM algorithm.

With the MMI method, the gray-scale intensities between the minimum and maximum values are linearly scaled, and joint histogram is created with same range histograms of sources. In this approach, we separate a SPCET image into three clusters (background, brain tissue, and focus, as shown in Fig. 3). The fuzzy map of brain tissue includes most geometric structure of brain, which can be used for MMI registration. Besides, the fuzzy map of focus can also be used for image fusion, because the focus map is the high radiative area and has the most interesting information for physicians.

\subsection{Maximum of Mutual Information (MMI)}

Mutual Information (MI) is developed from information theory, that is applied in the context of image registration to measure the amount of information that one image contains about the other. It is usually used for a measure of similarity for image registration, and belongs to voxel similarity based method. When images are registering, one image is nailed, and the other is shifted, translated, or scaled by a transformation. The MI measure in the two images is maximized at registration and it is based on entropy, which can be considered to describe the histogram dispersion of the image pixels' intensity. Usually, MI is defined as Eq. (6).

$$
M I(A ; B)=H(A)+H(B)-H(A ; B)
$$

where $\mathrm{H}(A)$ and $\mathrm{H}(B)$ are the entropies of the images $A$ and $B$, and $H(A ; B)$ is their joint entropy. The joint entropy is given by

$$
H(A ; B)=-\sum_{a=1}^{x} \sum_{k=1}^{N} p(a ; b) \log p(a ; b)
$$

$p(a ; b)$ is the probability of the co-occurrence of gray value $a$ in image $A$ and gray value $b$ in image $B$ at the same coordinate positions, named the joint probability distribution. When the images are aligned, the joint probability should be less than the case of any misalignment. 


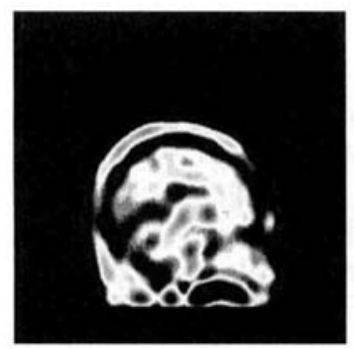

(a) brain tissue

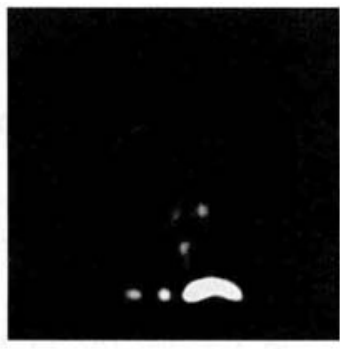

(b)focus
Fig. 3 The fuzzy map of a SPECT image

\section{EXPERIMENTAL RESULTS}

In the experiments, MR images used are T2weighted image with a TR of $4000 \mathrm{msec}$ and TE of 90 msec, slice thickness is $3 \mathrm{~mm}$, and image's matrix size is $256 \times 256 \times 36$ voxels. And SPECT images are Tc$99 \mathrm{~m}$ labeled tropanes of TRODAT, $128 \times 128 \times 64$ voxels, and each voxel is a $2.9 \mathrm{~mm}^{3}$ cubic. In our case, the fused images can aid physicians to analyze the quantity contained of dopamine in putamen and caudate nucleus for the diagnosis of Parkinsion's disease. In fact, this scheme is not only useful for Parkinson's disease, but also Alzheimer's disease, epilepsy or other brain degenerative diseases rely on $M R$ and SPECT images for clinical diagnosis, evaluation, and treatment.

To compare the performance of the proposed improved MMI and traditional MMI, three affinedinvariants including area, long-axis, and short-axis are employed to calculate error functions of both slice set of joint object.

The three error functions of area, long-axis and short-axis are listed in Eqs. (8)-(10):

$$
\begin{aligned}
& e_{\text {area }}=\frac{1}{S} \sum_{s_{1}}^{S}\left|\frac{\operatorname{area}\left(a_{s_{t}}\right)}{\max (\operatorname{area}(a))}-\frac{\operatorname{area}\left(b_{s_{t}}\right)}{\max (\operatorname{area}(b))}\right| \\
& e_{\text {long }}=\frac{1}{S} \sum_{s_{1}}^{S}\left|\frac{\operatorname{long}\left(a_{s_{i}}\right)}{\max (\operatorname{long}(a))}-\frac{\operatorname{long}\left(b_{s_{i}}\right)}{\max (\operatorname{long}(b))}\right|
\end{aligned}
$$

$e_{\text {short }}=\frac{1}{S} \sum_{s_{1}}^{S}\left|\frac{\operatorname{short}\left(a_{s_{i}}\right)}{\max (\operatorname{short}(a))}-\frac{\operatorname{short}\left(b_{s_{i}}\right)}{\max (\operatorname{short}(b))}\right|$

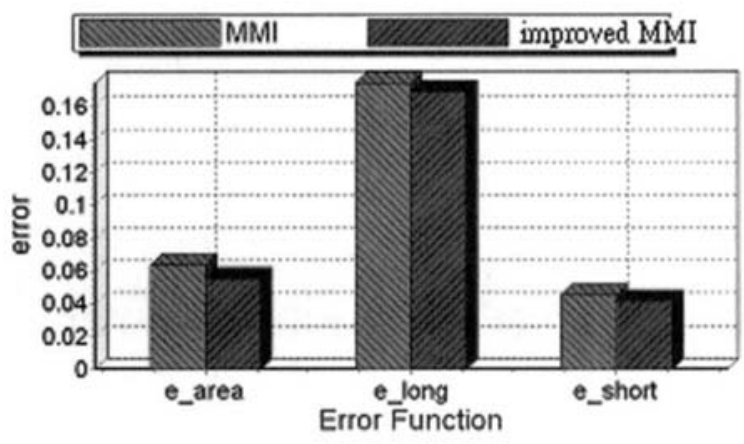

Fig. 4 The error functions of improved MMI and traditional MMI

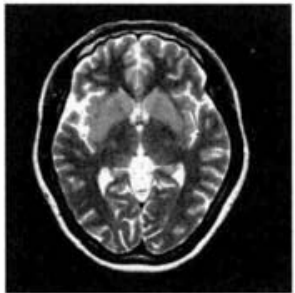

(a) 2-D display

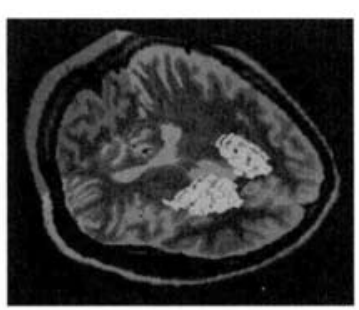

(b) 3-D display
Fig. 5. The 2-D and 3-D display of the fused images

where $S$ is total slice number of joint, $s_{i}$ is the slice sequence of image sets $a$ and $b . \max ()$ denotes the maximum value of whole image set. And area(), long(), and short) represent values of area, long-axis, and short-axis, respectively.

From Fig. 4, it is observed that the error functions of improved MMI are smaller than traditional MMI totally. Besides, Fig. 5 shows the fused images, which are displayed in 2-D and 3-D form. The color areas in Fig. 5(a) and (b) are reconstructed from the focus fuzzy map which is generated with the modified FCM as described in section 2.2. In fact, in the proposed scheme, enhanced-FCM not only provides fuzzy map for registration, but also plays a great role of interesting target segmentation.

\section{CONCLUSIONS}

In this approach, we have proposed an image fusion scheme, named improved MMI to solve the fusion problem of MR and SPCET images. By using IMSP algorithm to find the mid-sagittal plane of each image set, and Enhanced-FCM to effectively extract 
brain tissue map from SPECT image, this method can save large computing cost for MMI. The experimental results, using MR and SPECT images, to confirm the supcrior performance of the proposed method in comparison with the traditional MMI method are also included.

\section{ACKNOWLEDGEMENT}

The authors would like to thank the National Science Council of the R.O.C. for financial support of this manuscript under contract No. NSC92-2212-E182-002.

\section{REFERENCES}

1. F. Maes, D. Vandermelen, and P.Suetens, "Medical Image Registration Using Mutual Information", Proceedings of the IEEE, vol. 91, no. 10, pp.1699$1722,2003$.

2. P. Viola, W. Wells," Alignment by maximization of Mutual Information," Proceedings of the fifth International Conference on Computer Vision, pp. 15-25, 1995.

3. F. Maes, A. Collignon, D. Vandermelen, G. Marchal, and P. Suetens, "Multi-modality image registration by maximization of mutual information," IEEE Trans. Med. Imaging 16, pp. 187-198, 1997.

4. C. R. Meyer, J. L. Boes, B. Kim, P. H. Blad.,R. Zasadny, P. V. Kison, K. Koral, K. A. Frey, R. L. Wahl, "Demonstration on accuracy and clinical versatility of mutual information for automatic multi-modality image fusion using affine and thinplate spline warped geometric deformations, Med. Image Analysis, vol. 1, pp. 195-206, 1997.
5. J. B. A. Maintz, E. W. H. Meijering, M. A. Viergever, " general multimodal elastic registration based on mutual information", Medical Imaging, SPIE Press, vol. 3338, pp. 144-154, 1998.

6. T. Gaens, F. Maes, D. Vandermelen, P. Suetens, " Non-rigid multimodal image registration using mutual information", Medical image computing and computer-assisted intervention, Lecture Notes in Computer Science, vol. 1496, pp. 1099-1106, 1998.

7. Yanxi Liu, R.T. Collins, and W.E. Rothfus, "Robust Midsagittal Plane Extraction from Normal and Pathological 3-D Neuroradiology Images", IEEE Transactions on Medical imageing, vol.20, no.3, pp. 175-192, 2001.

8. Y.W. Lim and S.U. Lee, "On the color image segmentation algorithm based on the thresholding and the fuzzy c-means techniques," Pattern Recognition, vol. 23, pp. 935-952, 1990.

9. J. Suckling, T. Sigmundsson, K. Greenwood, and E.T. Bullmore, "A modified fuzzy clustering algorithm for operator independent brain tissue classification of dual echo MR images," Magnetic Resonance Imaging, vol. 17, pp. 1065-1076, 1999.

10. W.E. Phillips, R.P. Velthuizen, S. Phuphanich, L.O. Hall, L.P. Clarke, and M.L. Silbiger, "Application of fuzzy c-means segmentation technique for differentiation in MR images of a hemorrhagic glioblastoma multiforme," Magnetic Resonance Imaging, vol. 13, pp.277-290, 1995. 